\title{
Magnetic properties of bilayer graphene quantum dots in the presence of uniaxial strain
}

\author{
J. S. Nascimento, ${ }^{1,2,{ }^{*}}$ D. R. da Costa,,$^{1,3, \dagger}$ M. Zarenia, ${ }^{3, \ddagger}$ Andrey Chaves,,${ }^{1,}$ and J. M. Pereira Jr. ${ }^{1, \|}$ \\ ${ }^{1}$ Departamento de Física, Universidade Federal do Ceará, Caixa Postal 6030, Campus do Pici, 60455-900 Fortaleza, Ceará, Brazil \\ ${ }^{2}$ Departamento de Física, Universidade Federal do Piauí, 64049-550 Teresina, Piauí, Brazil \\ ${ }^{3}$ Department of Physics, University of Antwerp, Groenenborgerlaan 171, B-2020 Antwerp, Belgium
}

(Received 31 May 2017; published 18 September 2017)

\begin{abstract}
Using the tight-binding approach coupled with mean-field Hubbard model, we theoretically study the effect of mechanical deformations on the magnetic properties of bilayer graphene (BLG) quantum dots (QDs). Results are obtained for AA- and $\mathrm{AB}$ (Bernal)-stacked BLG QDs, considering different geometries (hexagonal, triangular and square shapes) and edge types (armchair and zigzag edges). In the absence of strain, our results show that (i) the magnetization is affected by taking different dot sizes only for hexagonal BLG QDs with zigzag edges, exhibiting different critical Hubbard interactions, and (ii) the magnetization does not depend on the interlayer hopping energies, except for the geometries with zigzag edges and AA stacking. In the presence of in-plane and uniaxial strain, for all geometries we obtain two different magnetization regimes depending on the applied strain amplitude. The appearance of such different regimes is due to the breaking of layer and sublattice symmetries in BLG QDs.
\end{abstract}

DOI: 10.1103/PhysRevB.96.115428

\section{INTRODUCTION}

A plethora of unique physical properties observed in graphene-based materials has generated a great interest on these materials, particularly owing to their potential for novel technological applications [1,2]. Many of these studies have been carried out to understand the electronic, magnetic, and mechanical properties of graphene nanostructures, such as nanoribbons, quantum dots (QDs) and quantum rings (QRs) [3-9]. One of the motivations concerning the investigation of these quantum confinement structures is related to the need of producing graphene-based system with an energy gap, which is generally absent in a pristine graphene sheet, and to the expectation that finite-size effects may significantly modify the graphene properties as result of the size quantization. The size, shapes, and types of edges of these graphene nanostructures have been found to strongly affect their intrinsic electronic properties, allowing for instance to control their energy gap [8-22].

Recently, it has been shown that graphene nanostructures can exhibit magnetic ordering, besides presenting promising features as long spin relaxation time and high electron mobility, which are advantageous for spintronic applications and sensor devices $[7,8,21,23-26]$. The nature of the magnetic ordering in graphene nanostructures is mainly related to the imbalance of sublattice atoms that might, e.g., give rise to a band of degenerate states near the Fermi energy level. Furthermore, it has been shown that the magnetic properties of graphenebased nanostructures exhibit different features from ordinary graphene due to the existence of edge states. These states are localized at the zigzag edges, decay quickly in the bulk and lead to a spin polarization of the ground state. Whereas

\footnotetext{
*jonasnascimento@ufpi.edu.br

†diego_rabelo@fisica.ufc.br

${ }^{\ddagger}$ mohammad.zarenia@uantwerpen.be

§andrey@fisica.ufc.br

"pereira@fisica.ufc.br
}

bulk graphene is a diamagnetic semimetal, zigzag graphene nanoflakes may have either ferromagnetic or antiferromagnetic ordered ground states, while armchair graphene nanoflakes are always nonmagnetic. It has been previously reported in the literature that the number and properties of edge states are sensitive to the geometry of the graphene QD and QR, such that geometry and edge type play an import role in the diamagnetic response of the graphene nanostructures [3,7-21,27-31].

Besides the application of external electric or magnetic fields, or by the controlling of the size, shape, edge type and number of layers [7,32-38], it has been also demonstrated that geometrical deformations can be used to tune the electronic and magnetic properties of the graphene nanostructures [39-46]. The main interest in its strain engineering is due to graphene's ability to withstand large mechanical stress, sustaining elastic strains up to $25 \%$ [47]. It has been shown that the effect of mechanical deformations on the graphene lattice is to change the hopping energies of the carbon atoms and to modify the band structure by shifting the Dirac cones with respect to each other [43-45]. In addition, previous works have investigated the effect of uniaxial strain on the optical [41] and magnetic [39,40] properties of graphene QDs using tight-binding and mean-field calculations, respectively. These studies revealed that magnetism can be enhanced up to $100 \%$ for strain values on the order of $20 \%$ for some dot geometries. Another type of QD, similar to the ones made of monolayer graphene, can be achieved by considering small flakes of bilayer graphene (BLG). In such BLG QDs, it has been recently shown that the edges and geometries play an important role and modify strongly the energy spectrum $[8,14-16,18,19]$, similar to monolayer QDs. Despite the considerable number of theoretical studies pertinent to the magnetic properties of monolayer graphene QDs, similar studies in BLG have been limited to triangular BLG flakes without taking in account the effect of strain [48]. Therefore a naturally related question arises, namely, how the magnetic properties of BLG QDs are modified by external strain. Systematic studies of magnetism in BLG flakes with different geometries and boundaries in the presence of strain are, to our knowledge, absent. 
In this paper, by combining the tight-binding (TB) approximation and the electron-electron interactions treated self-consistently at the level of the mean-field Hubbard model, we numerically explore how the magnetic ordering in BLG QDs with different geometries, stacking and edges, is affected by uniaxial strain. The paper is organized as follows. In Sec. II, we introduce the theoretical model used in our numerical calculations. In Sec. III, we discuss the self-consistent meanfield results obtained in the absence (Sec. III A) and in the presence (Sec. III B) of an applied strain. Finally, we summarize our results in Sec. IV.

\section{THEORETICAL MODEL}

In order to study the magnetic properties of BLG QDs, we use the widely applied one-orbital mean-field Hubbard model $[7,8,21,22,25,27-40]$. It consists in a $\pi$-orbital tight-binding model $\left(\mathcal{H}_{T B}\right)$ for single particles combined with the Hubbard model $\left(\mathcal{H}_{U}\right)$ to describe the repulsive electron-electron Coulomb interactions. The Hubbard-model Hamiltonian can be written as

$$
\mathcal{H}=\mathcal{H}_{T B}+\mathcal{H}_{U},
$$

where $\mathcal{H}_{T B}$ is the noninteracting term represented by the nearest-neighbor tight-binding Hamiltonian, given by

$$
\mathcal{H}_{T B}=\sum_{i, \sigma} \epsilon_{i \sigma} c_{i \sigma}^{\dagger} c_{i \sigma}+\sum_{i \neq j, \sigma}\left(\tau_{i j \sigma} c_{i \sigma}^{\dagger} c_{j \sigma}+\text { H.c. }\right),
$$

where $c_{i \sigma}\left(c_{i \sigma}^{\dagger}\right)$ annihilates (creates) an electron in site $i$ with spin $\sigma$ and on-site energy $\epsilon_{i \sigma}$. The sum is taken only between the nearest-neighbor sites $i$ and $j$, with hopping energy $\tau_{i j \sigma}$. In AA-stacked BLG, the atoms in the upper and lower layers are located directly on top of each other, whereas in an AB-stacked BLG the atoms in the A (B) sublattice from the bottom layer are coupled with B (A) atoms in the top layer (i.e., the two monolayers are shifted with respect to each other), as sketched in the insets of top and bottom panels of Fig. 1 for AA-staked and AB-staked BLG QDs, respectively [49]. The hopping energy between the atoms in the same layer is $\tau_{i j \sigma}=t=-2.8 \mathrm{eV}$, while the hopping for neighbor atoms stacked right on top of each other in different layers is, unless otherwise explicitly stated, $\tau_{i j \sigma}=t_{\perp}^{A B}=-0.4 \mathrm{eV}$ (for AB-stacked BLG QDs) and $\tau_{i j \sigma}=t_{\perp}^{A A}=-0.2 \mathrm{eV}$ (for AAstacked BLG QDs) $[14-19,48,49]$. We assume that $\epsilon_{i \sigma}=0$, resulting in an electron-hole symmetry for the energy spectrum obtained by the $\mathcal{H}_{T B}$ matrix, which is thus symmetric with respect to zero energy.

The Hubbard repulsion, treated in the mean-field approximation, in order to include the effect of electron-electron interaction, reads

$$
\mathcal{H}_{U}=U \sum_{i}\left(\left\langle n_{i \downarrow}\right\rangle n_{i \uparrow}+\left\langle n_{i \uparrow}\right\rangle n_{i \downarrow}\right),
$$

where $U(U>0)$ is called as the Hubbard parameter and denotes, in the short-range regime, the on-site Coulomb repulsion energy for each pair of electrons with opposite spins on the same site $i . n_{i \sigma}=c_{i \sigma}^{\dagger} c_{i \sigma}$ is the number operator and $\left\langle n_{i \sigma}\right\rangle$ is the average electron occupation number for spin-up $(\sigma=\uparrow)$ and spin-down $(\sigma=\downarrow)$ electrons.

In order to solve the problem for $\mathcal{H}$, we perform selfconsistent calculations, starting from a randomly chosen initial distribution of the average electron occupation number $\left\langle n_{i \sigma}\right\rangle$ and then, by diagonalizing the Hamiltonian (1), we obtain the new eigenvalues and eigenvectors that are used to compute the updated spin densities for the next iteration. This procedure is repeated iteratively until the spin density as well as the eigenvalues of $\mathcal{H}$ converge. To be more specific, the criterion of convergence of the self-consistency is reached when the maximum change of the spin density over the atomic sites drops below $\eta$, a convergence parameter chosen ranging from $\eta=10^{-9}$ to $10^{-15}$, depending on the analysed case. Accordingly, with the self-consistent achieved spin densities, one can compute the magnetic moment per atomic site

$$
m_{i}=\left(\left\langle n_{i \uparrow}\right\rangle-\left\langle n_{i \downarrow}\right\rangle\right) / 2,
$$

the total spin $S=\sum_{i} m_{i}$, the maximum of magnetization $m_{\max }$, the charge distribution $\left\langle n_{i \uparrow}\right\rangle+\left\langle n_{i \downarrow}\right\rangle$, as well as the energy spectrum $E_{n, \sigma}$. These magnetic properties will be discussed in the next section for different parameters of BLG QDs. It is important to emphasize that the BLG QDs studied

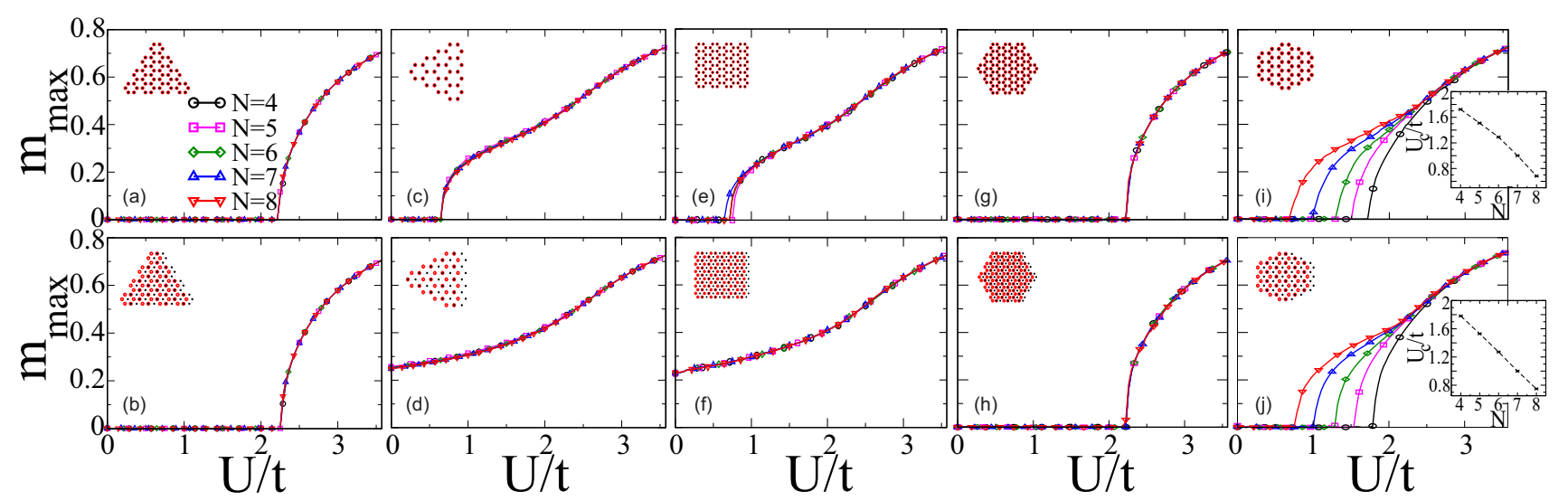

FIG. 1. Variation in the magnetization as function of the Hubbard interaction $U / t$ for different dot size $N$ and for triangular [(a)-(d)], square [(e) and (f)], and hexagonal [(g), (h), (i), and (j)] BLG QDs with armchair [(a), (b), (g), and (h)] and zigzag [(c), (d), (i), and (j)] edges. The top (bottom) panels correspond to (AB-)AA-stacked BLG. The insets in (i) and (j) represent the critical Hubbard interaction as a function of the dot size. The interlayer hopping energies are $t_{\perp}=0.2$ and $0.4 \mathrm{eV}$ for AA- and AB-stacked BLG QDs. 
here with hexagonal and triangular shapes are characterized by the number of carbon hexagons $(N)$ in each dot side and we consider only symmetric dots. Thus all dot sides have the same length and the number of carbon atoms at the top and bottom layers are the same. The only exception is the square dots, where the number of carbon rings along the zigzag direction is different (greater) than the number along the armchair direction, in order to preserve the length of the dot sides to be approximately the same for all sides, since square dots have two zigzag edges and two armchair edges. In this case, we take $N$ for the armchair direction.

An important point is the range of physical meaningful magnitudes for $U$ in Eq. (3). To our knowledge, there is currently no consensus on the actual values of $U$ for the case of graphene [7]. This is due to current absence of standard and direct experiments performed on magnetic graphene systems which would allow to estimate $U$. In a recent study of magnetism in disordered graphene and irradiated graphite [50], was reported for the $U$ parameter to lie in the range $U \approx 3.0-3.5 \mathrm{eV}$. In this study, the accepted interval obtained from magnetic resonance measurements in transpolyacetylene (a one-dimensional $\mathrm{sp}^{2}$ carbon system that resembles a shortwidth zigzag graphene nanoribbon) was considered [51,52]. As examples of the aforementioned absence of a general consensus about the value of $U$, recent works in graphene confinement structures consider $U=5.6$ [28], 1.5 to 3.5 [30], 4.158 [35], 2.0 to 3.5 [39], 2.8 to 8.4 [40], 5.6 to $11.2 \mathrm{eV}$ [46] for graphene QDs and $U=3.24$ [31], 2.82 [32], 2.7 [33], and $2.75 \mathrm{eV}$ [34] for graphene QRs.

A recent comparison between results for the magnetic properties of graphene QDs [30] and ribbons [22,26] obtained from the mean field Hubbard model and from first-principle calculations found a good agreement if the Hubbard parameter $U$ is chosen appropriately. Moreover, the mean-field Hubbard model is found to capture the low-energy physics of graphene nanoislands [30] and nanoribbons [22,26], showing that next-to-nearest neighbor hoppings, long-range Coulomb interactions, and correlations included in the first-principle calculations have a minor effect on the physical properties in the low-energy regime. However, it is important to mention that the long-range Coulomb interactions and correlations have a minor effect, since this system is charge neutral with a homegeneous charge distribution. On the other hand, away from local charge neutrality, as for instance in the presence of bias potential in the layers, the long-range Coulomb interactions and correlations may become significant. Thus, this ensures the validity of Hubbard model to the present study of magnetic properties in BLG QDs.

\section{NUMERICAL RESULTS}

\section{A. In the absence of strain}

First, let us investigate the magnetic ordering for unstrained BLG QDs with different geometries and edge types. In Fig. 1, we depict the dependence of the maximum value of magnetization $m_{\max }$ as a function of the on-site Coulomb Hubbard repulsion $U / t$ for different numbers of carbon rings $N$ in each dot side. Top and bottom panels correspond to results for AA- and AB-staked BLG QDs, respectively, considering armchair [(a), (b), (g), and (h)] and zigzag [(c), (d), (i), and (j)] edges. Notice that, in general, irrespective to the size of the dot, the magnetic ordering for the triangular, Figs. 1(a)-1(d), and square, Figs. 1(e) and 1(f), BLG QDs with zigzag or armchair edges and with AA- or AB-stacking, as well as for hexagonal BLG QDs with armchair edges with both stacking types, Figs. 1(g) and 1(h), shows to be independent of $N$, without any considerable variation with the size. However, the same feature does not hold for hexagonal BLG QDs with zigzag edges with both stackings [see Figs. 1(i) and 1(j)]. The nature of the size-dependent magnetization for zigzag-edged hexagonal BLG QDs, Figs. 1(i) and 1(j), is linked to its shape and its zigzag edges. In hexagon, three edges contain sites belonging only to the $A$ sublattice ( $A$ type) and the other three are $B$ type, such that one has edge and sublattice balances implying in a global balance. According to Ref. [30], there is a competition, in the case of zigzag hexagons, between the dispersion of the single-particle spectra and interactions, where the dispersion occurs because of the hybridization of states that otherwise would lie in a single sublattice close to the edge. As a result, smaller nanostructures feature larger hybridization and are less prone to develop magnetic order, explaining the reason why $U_{c}$ for smaller QDs is higher than the larger ones, as shown in the insets of Figs. 1(i) and 1(j). Moreover, as a consequence of the compensated lattice, the spin density for three edges point towards up direction, whereas the other three edges points towards the opposite direction (the magnetization spin density for hexagonal BLG QDs will be discussed in more detail further on).

Previous works on monolayer graphene QDs have reported a similar size-dependence of the QDs magnetization as compared to the ones obtained here for BLG QDs. FernándezRossier and Palacios [30] have found a robustness in the results of magnetization with respect to the value of $U$ for triangles with $N$ between 5 and 30, while Viana-Gomes et al. [39] have shown that $m_{\max }$ as a function of $U$ for square and hexagonal armchair QDs do not display noticeable variation with the size. Furthermore, the magnetization for triangular, Figs. 1(a) and 1(b), and hexagonal, Figs. 1(g) and 1(h), BLG QDs with armchair edges are equivalent to the graphene ordinary system, exhibiting a second-order phase transition at critical on-site Coulomb Hubbard interaction $U_{c} \simeq 2.2 t \simeq 6.12 \mathrm{eV}$ $[7,30,39]$. The reasons why the second-order transition occurs similarly to infinite graphene are due to the absence of zigzag edges in these geometries and also because these QDs possess sublattice symmetry with a balanced lattice such that the number of atoms in the sublattice $A$ and $B$ in each layer is the same. Above the critical on-site Coulomb repulsion $U_{c}$, an antiferromagnetic ordering appears as a consequence of the bipartite nature of the honeycomb lattice [28]. On the other hand, the geometries with zigzag edges exhibit a smaller value of $U_{c}$ [see Figs. 1(c), 1(e), 1(i), and 1(j) for AA-stacked triangular, AA-stacked square and AA- and AB-stacked hexagonal shapes, respectively], which is related to the imbalanced structure with different number of atoms belonging the sublattices $A$ and $B$. One observes that the slope of the magnetization curves for triangular zigzag-edged, Figs. 1(c) and 1(d), and square, Figs. 1(e) and 1(f), BLG QDs are approximately the same, which can be interpreted as a boundary effect, determined by the zigzag terminations 


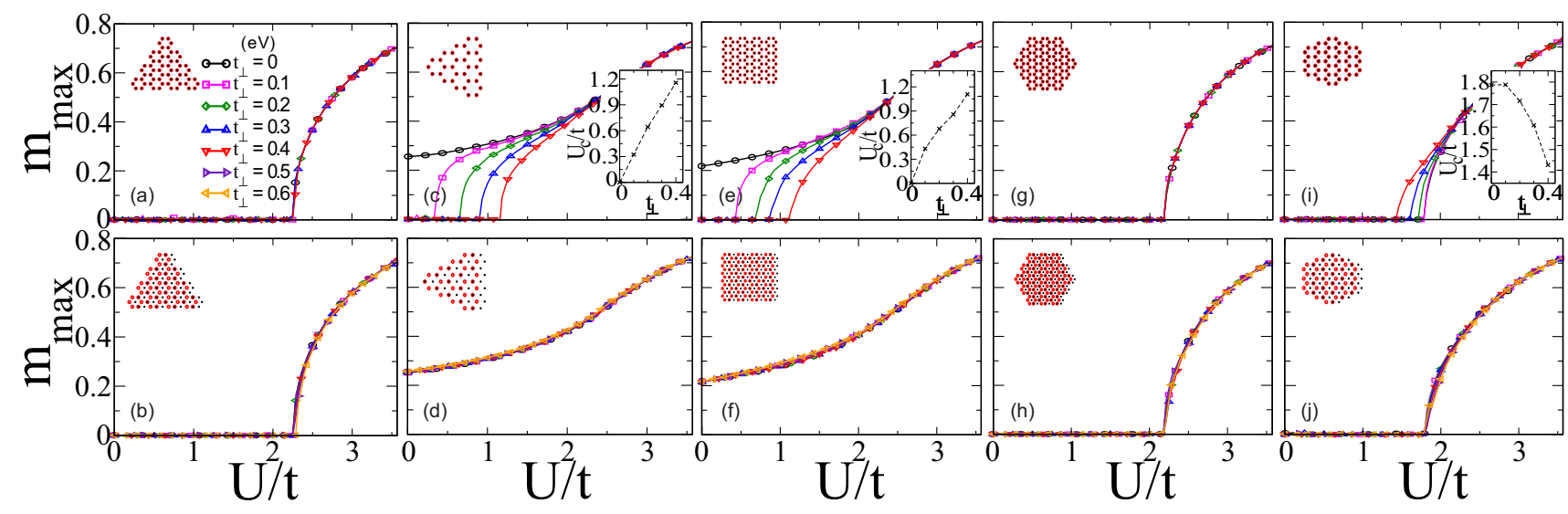

FIG. 2. The same as in Fig. 1, but now considering different interlayer hopping energies $t_{\perp}$. It was assumed $N=4$ for all geometries. The insets in (c), (e), and (i) represent the critical Hubbard interaction as a function of $t_{\perp}$.

in both geometries that play a predominant role in their magnetizations. Besides, AB-stacked triangular zigzag-edged and $\mathrm{AB}$-stacked square BLG QDs present finite magnetization for any finite $U$, being a consequence of the presence of zigzag edges and the layer symmetry in these QDs. This point will become more clear further through the discussion of the density probability for these structures.

Taking different interlayer distances leads to different interlayer hopping energies between the atoms belonging to the different layers. Therefore an analysis of variation of the interlayer hopping energy around the actual value $t_{\perp}=0.2$ and $0.4 \mathrm{eV}$ for $\mathrm{AA}$ - and AB-stacked bilayers, respectively, allows us to check in which cases coupled layers would show different results from the decoupled ones and to verify the robustness of $m_{\max }$ with respect to $t_{\perp}$ for different dot shapes and $U$ values. Thus Fig. 2 shows the magnetization as a function of the Hubbard interaction $U$ for different $t_{\perp}$ hopping energies ranging from $t_{\perp}=0$ to $0.6 \mathrm{eV}$, considering triangular, Figs. 2(a)-2(d), square, Figs. 2(e) and 2(f), and hexagonal, Figs. 2(g)-2(j), BLG QDs with AA (top panels) and $\mathrm{AB}$ (bottom panels) stacking. In our calculations, we fixed the interatomic distance between the atoms in the same layer $(t=-2.8 \mathrm{eV})$ and the system size $(N=4)$. Our theoretical results show that the maximum value of magnetization does not depend on the $t_{\perp}$ hopping energies for all studied ABstacked BLG QDs with both zigzag and armchair edges (bottom panels) and AA-stacked BLG QDs with only armchair terminations, Figs. 2(a) and 2(g). Therefore the two decoupled layers (for $t_{\perp}=0$ ) have the same magnetization as the coupled ones for almost all geometries. Consequently, the presence of any defect in a nanostructured BLG system whose only effect is to reduce or to increase the coupling energies between the two layer, will not significantly affect the mean-field results. However, this is not the case for AA-stacked triangular [Fig. 2(c)], square [Fig. 2(e)], and hexagonal [Fig. 2(i)] BLG QDs with zigzag edges. The reason is related to the boundary effect (zigzag edges) and because the magnetic moments of the two layers in AA-stacked BLG QDs with zigzag edges are coupled antiferromagnetically (for $U>U_{C}$ ) as $t_{\perp}$ increases. In fact, the spin-resolved magnetic moment for the atoms in the top layer points towards to opposite direction with respect to the underneath atoms at the bottom layer and thus the layers are strongly affected by the perpendicular hopping energy changes (that becomes clearer later from the results for the spin densities). The insets of Figs. 2(c), 2(e), and 2(i) show the behavior of the critical on-site Coulomb Hubbard parameter for increasing interlayer coupling $t_{\perp}$. Notice that imbalanced structures with different numbers of atoms per sublattice, Fig. 2(c) triangular QD with zigzag edges and in the Fig. 2(e) square QD, exhibit larger $U_{c}$ values, whereas balanced structures, Fig. 2(i) hexagonal QD with zigzag edge, i.e., $N_{A}=N_{B}, U_{c}$ decreases as a function of $t_{\perp}$ [as in Fig. 1(i)]. Therefore, for these QDs, as we will show later by analyzing the spin densities, one can switch from an ordering when both layers have the same spin orientations, behaving as two isolated monolayer graphene QDs, to a coupling with antiferromagnetic ordering, simply by changing the distance between the layers.

It is worth mentioning that the amplitude of magnetization $m_{\max }$ obtained here for all studied BLG QDs (Figs. 1 and 2) were approximately the same values reported for monolayer graphene QDs by taking fixed system parameters $(N, t)$ and Hubbard term $(U)[28,39]$. It is a consequence of the fact that $m_{\max }$ is computed by taking into account the magnetic moment per atomic site, then for a fixed $U, m_{\max }$ is a local property and is independent of the number of atomic sites. Moreover, since a BLG system obeys the layer symmetry, i.e., the number of atoms in both layers is the same, one has double the number of atoms as compared to the monolayer system. Due to this layer symmetry and the doubled number of atoms in BLG, the total spin $S$ is doubled compared to monolayer QDs. This agrees with Lieb's theorem [53] that states that in the case of repulsive electron-electron interactions $(U>0)$, a bipartite system has the ground state characterized by the total spin [7]

$$
S=\frac{\left|N_{A}-N_{B}\right|}{2}
$$

where $N_{A}\left(N_{B}\right)$ is the number of atoms in the sublattice $A(B)$. According to Lieb's theorem and Eq. (5), it is also clear that the value of the total spin $S$ depends on the geometry, the edge type and the size of QD, since some geometries possess a balanced (imbalanced) lattice with the same (different) number of atoms in sublattices A and B, emerging in different values for $S$. 


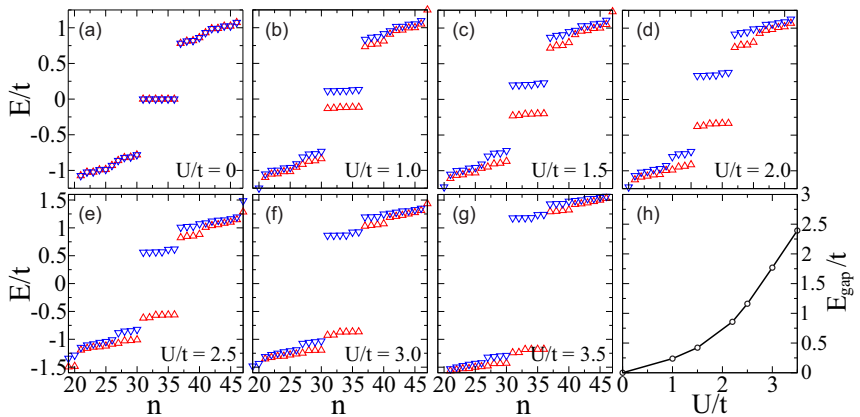

FIG. 3. (a)-(g) Evolution of the spin-resolved energy levels near the Fermi energy (set to zero) for a zigzag-edge triangular AB-stacked BLG QD with $N=4$ carbon hexagon in each side, by taking different values of the Hubbard interaction $U / t$. The interlayer hopping energy is $t_{\perp}=0.4 \mathrm{eV}$. The blue down (red up) triangles correspond to the spin-down (spin-up) energy levels. (h) Induced gap energy by the Hubbard interaction.

Figure 3 shows the evolution of the energy spectra for spinup (red up triangles) and spin-down (blue down triangles) states of a triangular BLG QD with zigzag edges and dot size $N=4$ by changing the Hubbard interaction range from $U / t=0$ to 3.5. Note that without considering the effect of onsite Coulomb repulsion $(U / t=0)$, the energy spectra exhibits electron-hole symmetry due to the bipartite lattice implying in a total spin equal to zero and there appears a degenerate set of zero-energy states that corresponds to the degenerate edge states at the Fermi energy $(E=0)$. The number of zeroenergy states is equal to $2(N-1)=6$, which is twice the value from monolayer graphene QD with triangular shape and zigzag edges [30,35], being related to the layer symmetry of the BLG system. This result is in agreement with previous work by Güçlï et al. [48] on the same geometry but with the triangles from the bottom and top layers having different numbers of atoms and thus, because the layer asymmetry, they obtained a different number of zero-energy states corresponding to the bottom and top layers. Note also that there is a large energy gap between the zero-energy edge states and the first nonzero state. Although not shown here, the latter are mostly spread along the QD. Considering $U / t \neq 0$, we verify that an energy gap opens around the $2(N-1)$-degenerate zero-energy states, spiting them in two groups of $(N-1)$-states for spin up and spin down [see Fig. 3(b)]. Moreover, the presence of Hubbard interaction term induces a nonzero total spin, $S=3$. As $U / t$ increases [see Figs. 3(b)-3(g)], the energy gap between the two groups of $(N-1)$-states increases, as emphasized in Fig. 3(e), while the total spin remains the same. Therefore the on-site Coulomb repulsion allows to control the relative position of the zero-energy states. In the next section, we will investigate how the presence of an uniaxial strain affects the magnetic properties of the BLG QDs.

\section{B. In the presence of strain}

In strained graphene, the interatomic distances change, thus modifying the hopping energies. The in-plane hopping parameter $\tau_{i j}$ in a free-standing layer can be transformed in
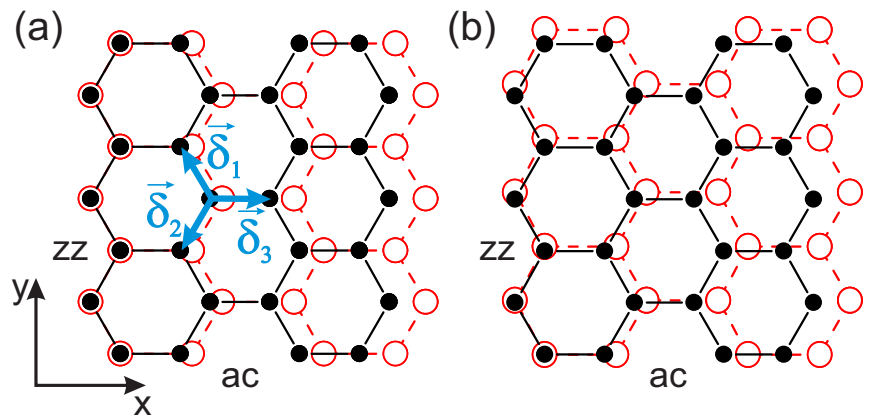

FIG. 4. Schematic representation of the effect of strain along $x$ direction (armchair) on the length of the nearest-neighbor carbon atoms (a) by keeping the atomic positions in $y$ direction unchanged, and (b) by considering an elastic deformation in both directions. The black solid (red open) symbols denote the carbon sites in the absence (presence) of strain and $\boldsymbol{\delta}_{l}$ (with $l=1,2$, and 3 ) are the lattice vectors.

the following form $[39,41,44]$ :

$$
\tau_{i j}^{\prime} \rightarrow \tau_{i j} e^{-3.37\left(\left|\delta_{i j}\right| / a_{0}-1\right)},
$$

with an explicit lattice-distance dependence, where $a_{0}=0.142$ $\mathrm{nm}$ is the unstrained intercarbon distance and $\boldsymbol{\delta}_{i j}$ is the distance vector between the adjacent atoms $i$ and $j$ in the strained lattice that is given, with respect to the undeformed lattice distances $\boldsymbol{\delta}_{i j}^{0}$, by

$$
\boldsymbol{\delta}_{i j}=(1+\boldsymbol{\epsilon}) \cdot \boldsymbol{\delta}_{i j}^{0} .
$$

$\boldsymbol{\epsilon}$ is the strain tensor in the lattice coordinate systems, given by

$$
\boldsymbol{\epsilon}=\epsilon\left(\begin{array}{cc}
\cos ^{2} \theta-v \sin ^{2} \theta & (1+v) \cos \theta \sin \theta \\
(1+v) \cos \theta \sin \theta & \sin ^{2} \theta-v \cos ^{2} \theta
\end{array}\right),
$$

where $\epsilon$ is the tunable strain modulus, $v$ is the Poisson ratio, and $\theta$ denotes the angle with respect to the zigzag direction ( $y$ direction in Fig. 4) along the applied strain direction. Applied tensions along the zigzag $(y)$ and armchair $(x)$ directions are considered by taking $\theta=\pi / 2$ and 0 in Eq. (8), respectively. For these two particularly interesting cases, the uniaxial inplane strain can be incorporated via Eq. (7) using the following deformed bond lengths:

$$
\left|\boldsymbol{\delta}_{l}\right|= \begin{cases}\left(1+\frac{3}{4} \epsilon-\frac{1}{4} \epsilon \nu\right) a_{0} & \text { for } l=1 \text { and } 2 \\ (1-\epsilon \nu) a_{0}, & \text { for } l=3\end{cases}
$$

for zigzag case and

$$
\left|\boldsymbol{\delta}_{l}\right|= \begin{cases}\left(1+\frac{1}{4} \epsilon-\frac{3}{4} \epsilon \mathcal{V}\right) a_{0} & \text { for } l=1 \text { and } 2 \\ (1+v) a_{0} & \text { for } l=3\end{cases}
$$

for armchair case. $\boldsymbol{\delta}_{l}$ 's with $l=1,2$, and 3, are shown with blue arrows in Fig. 4. In the elastic and linear deformation regime, strain along the zigzag direction makes the deformed bond lengths $\left|\boldsymbol{\delta}_{1}\right|$ and $\left|\boldsymbol{\delta}_{2}\right|$ extended and $\left|\boldsymbol{\delta}_{3}\right|$ contracted, while for the armchair case all three bonds become extended $[39,41,44]$. The value of the Poisson's ratio used for graphite and in some works also for graphene is $v=0.165[41,44]$, while recent works in graphene QDs has considered $v=0.3$ [39]. In order to make a direct comparison with the strained results in graphene QDs[39], we also assume $v=0.3$. 
Such uniaxial and elastic strain is expected to be attainable by using, e.g., flexible substrates [54], where the BLG QD is deposited on the flexible substrate and then one stretches the substrate by a mechanical device. It is important to point out that the interlayer hopping $t_{\perp}$ will remain constant, since we are considering pure in-plane lattice deformation and also because both coupled layers are subjected to the same strain amplitude. This ensures that the hopping related to $t_{\perp}$ will always be perpendicular to the bilayer sheets [19].

A sketch of the lattice deformation along the armchair $(x)$ boundary is illustrated in Fig. 4 for a very large value of $\epsilon$, in order to clearly show the lattice changes, where red open (black solid) symbols represent the (un)deformed graphene lattice. We will only show the results for the system under planar stress with tension along the armchair directions and keeping the atom positions in $y$ direction unchanged [Fig. 4(a)]. We have verified (although not shown here) that applied strain along the zigzag direction leads to qualitatively similar features as for the armchair case. We have also checked that elastic deformations like the one in Fig. 4(b), in which both edges deform, produce qualitatively and quantitatively similar results. This agreement between these results is due to the fact that the range for $\epsilon$ used here does not change significantly the atom positions in the $y$ direction. As reported in Refs. [39], a similar physical situation was also noted for monolayer graphene QDs, i.e., strained monolayer graphene QDs present qualitatively the same magnetization by deforming it along either the zigzag or armchair directions. That is also in agreement with recent results obtained by L. Wang et al. [55] for the elastic behavior of BLG, when deformed up to $20 \%$. For illustration purposes, all results in this section were obtained for $N=4$, since as shown in Fig. 1 the magnetization, in general, does not dependent on the different dot sizes, exhibiting similar features for all $N$ and thus ensuring us that we should have negligible finite-size effects.

Figure 5 presents the results for magnetization of BLG QDs with different geometries, edges and stacking as a function of the on-site Coulomb parameter $U$ in the presence of external in-plane deformation applied along the armchair direction. Top (bottom) panels correspond to the results for (AB-)AA-stacked BLG QDs for triangular [(a)-(d)], square [(e)-(f)], and hexagonal [(g)-(j)] shapes and with armchair $[(\mathrm{a}),(\mathrm{b}),(\mathrm{g})$, and $(\mathrm{h})]$ and zigzag $[(\mathrm{c}),(\mathrm{d}),(\mathrm{i})$, and $(\mathrm{j})]$ edges. By applying strain with modulus $\epsilon$ ranging from 0 to 0.2 , we notice that the global effect of uniaxial deformation is an enhancement of the magnitude of the magnetization $m_{\max }$, independent of the edge type and dot shape. However, a firstorder transition is seen with respect to the strain magnitude [as shown in the insets of Figs. 5(a)-5(j) for magnetization as a function of $\epsilon$ for a fixed value of $U / t]$, such that for all studied cases $m_{\max }$ is kept unchanged for $\epsilon \leqslant 0.7 \times 10^{-4}$, whereas it is enhanced for $\epsilon>0.7 \times 10^{-4}$, with the same magnetization values. Viana-Gomes et al. [39] have reported an increase in the maximum of the magnetization for square and hexagonal monolayer graphene QDs as $\epsilon$ increases. In case of monolayer QDs, the enhancement is not as abrupt and the modulus of strain $\epsilon$ required for an pronounced enhancement is approximately two orders of magnitude higher than for BLG. The reason for the low range of $\epsilon$ in strained BLG QDs is due to the fact that BLG system is more complex than the monolayer ones, regarding the fact that it admits more possibilities of magnetic ordering due to the correlation effect between the layers that are now coupled, being this way more sensitive to any deformation. For very small strain amplitudes, that implies that hopping energies should not be affected significantly by the deformation of the lattice, explaining the unchanged results for $\epsilon \leqslant 0.7 \times 10^{-4}$ that remain as the unstrained one for $\epsilon=0$ (black dashed lines). Experimentally, these small strain values $\left(\epsilon \leqslant 0.7 \times 10^{-4}\right)$ are difficult to be obtained, such that any applied tensile strength that is experimental feasible will result in a magnetization that matches on the top of the curve for $\epsilon=0.2$ (green triangle symbols), for example. Moreover, strain brings up an explicit variation in the critical Hubbard repulsion term $U_{c}$ for the BLG QD structures which have a finite $U_{c}$. For AA-stacked BLG QDs, top panels in Fig. 5 show that the applied in-plane strain reduces the value of $U_{c}$, not in a gradual way, as reported in Ref. [39] for hexagonal and square monolayer graphene QDs, but rather abruptly, such that $U_{c}^{\text {strained }}<U_{c}^{\text {unstrained }}$ for any strain amplitude higher than $\epsilon>$ $0.7 \times 10^{-4}$. For instance, $U_{c}^{\text {strained }} / t \approx 0.33<U_{c}^{\text {unstrained }} / t \approx$ 0.65 for triangular and $U_{c}^{\text {strained }} / t \approx 0.62<U_{c}^{\text {unstrained }} / t \approx$ 1.72 for hexagonal BLG QDs with zigzag edges, as shown
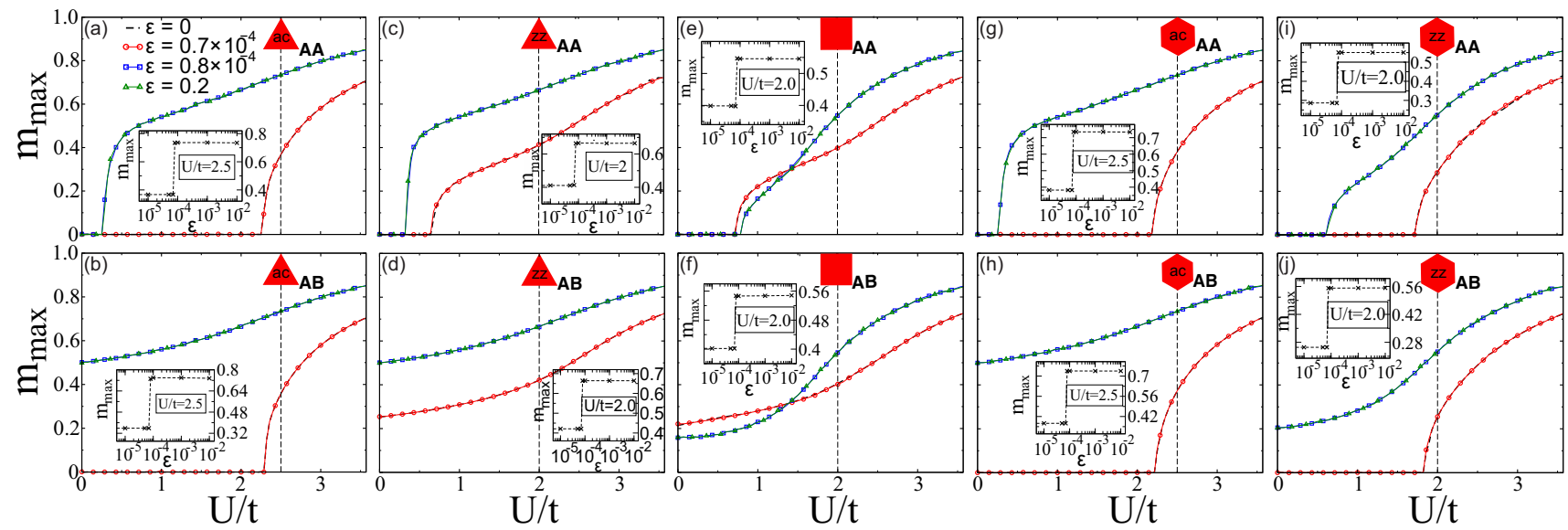

FIG. 5. The same as in Fig. 1, but now for different deformation amplitudes $\epsilon$, ranging from 0 to 0.2 . It was assumed $N=4$ for all geometries. The hopping parameter $t$ on the $x$ axis $(U / t)$ is the original unstrained one $|t|=2.8 \mathrm{eV}$. 

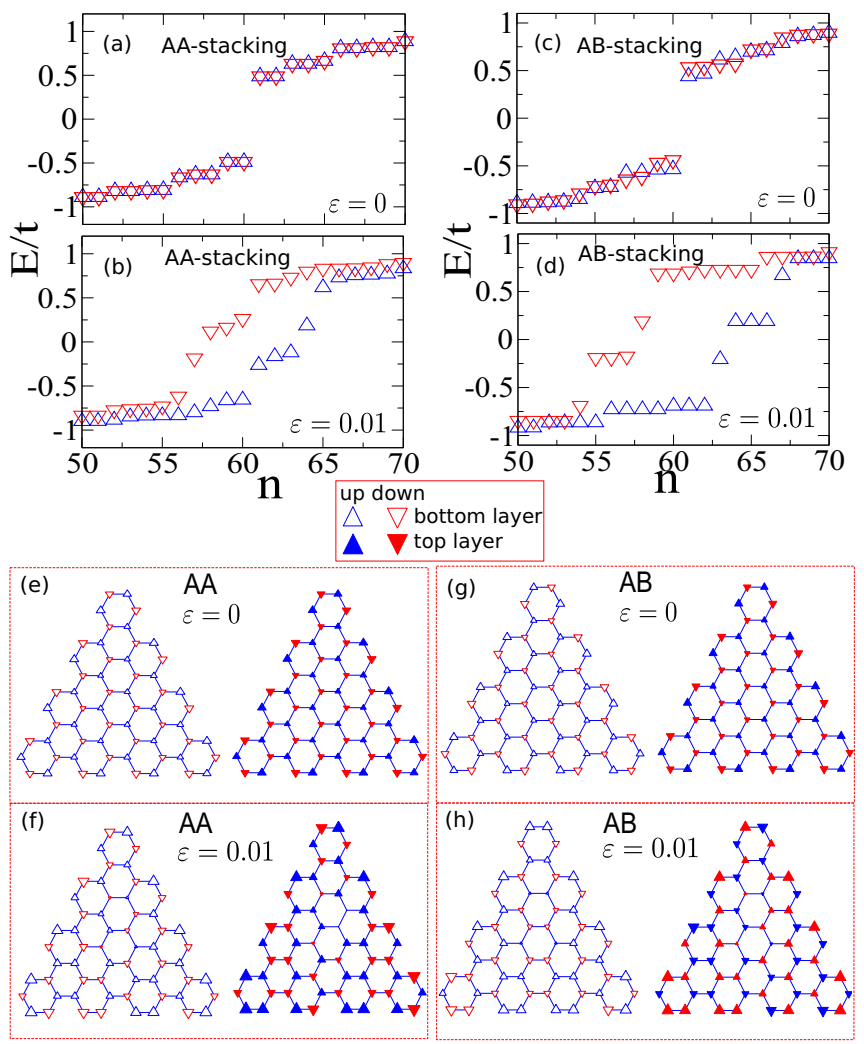

FIG. 6. (a)-(d) Spin-resolved energy levels near the Fermi energy (set to zero) and (e)-(h) spin density for armchair-edged triangular BLG QDs with strain amplitudes $\epsilon=0$ [(a), (c), (e), and (g)] and 0.01 [(b), (d), (f), and (h)] and AA [(a), (b), (e), and (f)] and AB [(c), (d), (g), and (h)] stackings. We assume Coulomb repulsion $U / t=2.5$. Blue up (red down) triangles correspond to the spin-up (spin-down) electrons in (a) to (d). Full (empty) symbols indicate top (bottom) layer in (e) to (h), and the size of the symbol is proportional to the spin polarization.

in Figs. 5(c) and 5(i), respectively. This way, the value of $U_{c}$ can be used to characterize the two different regimes of $m_{\max }$ for BLG QDs in the presence of strain.

Let us consider the decreasing of $U_{c}$ for strained AAstacked BLG QD structures. Although $U$ is a local (on-site) property, we observe that $U_{c}$ depends on the external stress. Keeping in mind that the hopping energies are strongly affected by changes in the interatomic distances, and that the results for bulk systems and armchair-edged QDs for $U_{c}$ are related to the uniform hopping parameter $t$ by $U_{c} \simeq 2.2 t$, as previously discussed, we have that a change in $t$ produces a change in the absolute value of $U_{c}$, which can be summarized in an explicit dependence of $U_{c}$ on the strain, $U_{c}(\epsilon) \simeq \alpha t(\epsilon)$, where $\alpha$ should be around the bulk value of 2.2. A similar discussion was presented in Ref. [39] for monolayer graphene QDs.

On the other hand, the effect of uniaxial strain in ABstacked BLG QDs leads to finite magnetization for any finite $U$. Thus for $U<U_{c}^{\text {unstrained }}$, these QDs switch from a nonmagnetic to a magnetically ordered state simply by applying strain. The pairs of Figs. 5(a)-5(g) and 5(b)-5(h) show very similar behavior for the maximum of magnetization for AA- and AB-stacked BLG QDs with armchair edges
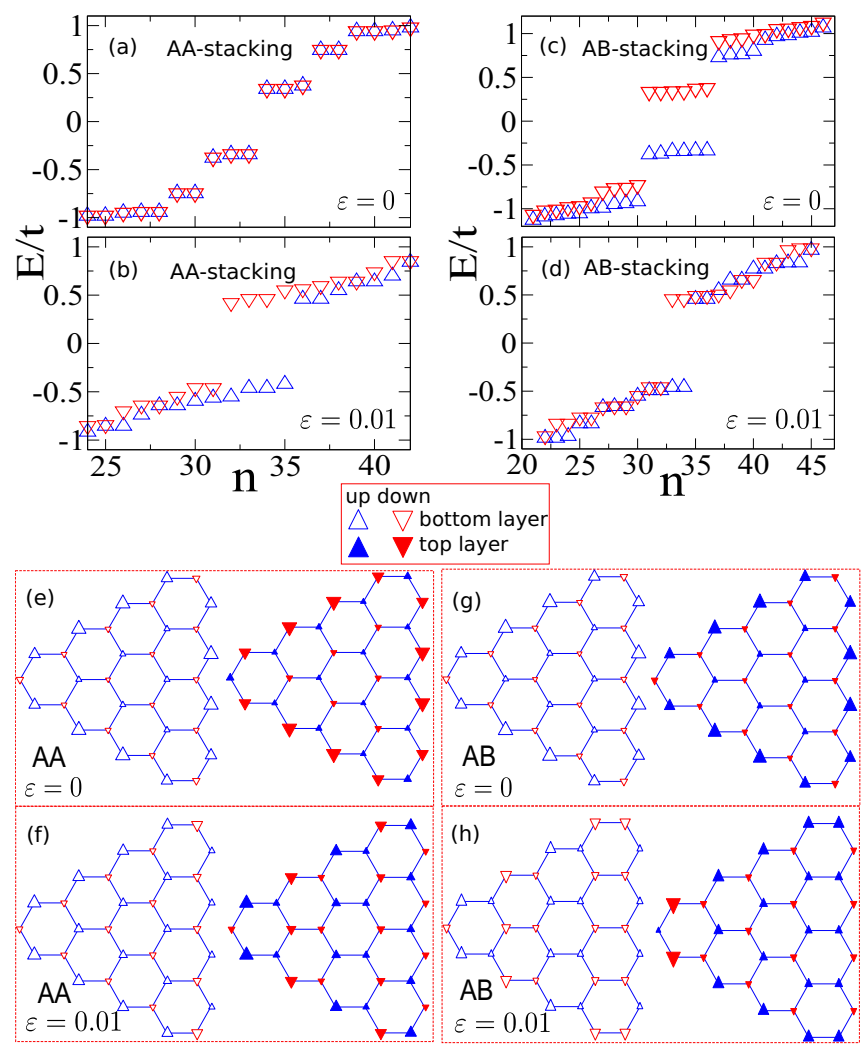

FIG. 7. The same as in Fig. 6, but now for triangular BLG QDs with zigzag edges and Coulomb repulsion $U / t=2.0$.

and triangular and hexagonal shapes $\left(U_{c}^{\text {strained }} / t \approx 0.25\right.$ for both armchair-edged triangular and hexagonal BLG QDs), as expected, since triangular and hexagonal dots with armchair edges exhibit similar magnetization independent of the dot size $N$ and interlayer hopping energy $t_{\perp}$, as seen in Figs. 1(a), 1(b), 1(g), 1(h) and 2(a), 2(b), 2(g), 2(h) for the unstrained BLG QDs. This is a consequence of the balance of these structures, having the same number of atoms belonging to different sublattices, and also due to the armchair boundaries. Also, that the maximum of magnetization for square BLG QDs with both $\mathrm{AA}$ and $\mathrm{AB}$ stacking exhibits a crossing between the strained and unstrained cases around $U / t \approx 1.42$. This can be interpreted as due to an effect of the interplay between the boundary effect and tensile strength, bringing up a competition between the zigzag edge states and the bulk states. This will be verified below by plotting the density functions of square BLG QDs with and without strain.

In order to understand the two different regimes found in the maximum of magnetization for strained and unstrained systems in Fig. 5, in Figs. 6 to 10, we show the spin-resolved energy levels [four top panels, (a) to (d)] and the spin density [four bottom panels, (e) to (h)] for triangular (Figs. 6 and 7), hexagonal (Figs. 8 and 9), and square (Fig. 10) BLG QDs with strain amplitudes $\epsilon=0[(\mathrm{a}),(\mathrm{c}),(\mathrm{e})$, and (g)] and 0.01 [(b), (d), (f), and (h)], and stacking AA (left panels [(a), (b), (e), and (f)]) and $\mathrm{AB}$ (right panels [(c), (d), (g), and (h)). From Figs. 6 to 10, one can clearly see that applied uniaxial strain dramatically modifies the spin-resolved energy levels and the spin densities for all studied BLG QDs. For instance, 

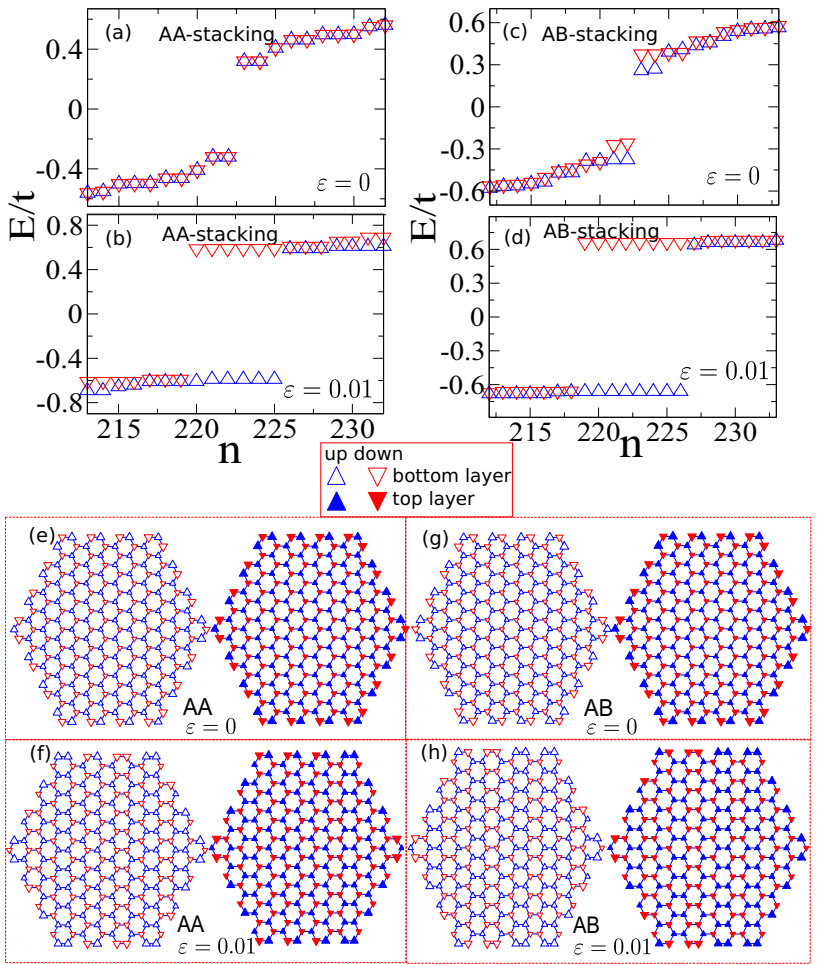

FIG. 8. The same as in Fig. 6, but now for hexagonal BLG QDs with armchair edges and Coulomb repulsion $U / t=2.0$.

note that in general, the effect of strain on the energy levels is either to lift the degeneracies, as observed for triangular (Figs. 6 and 7) and hexagonal (Figs. 8 and 9) BLG QDs, or to group the energy levels and thus increase the degeneracy,
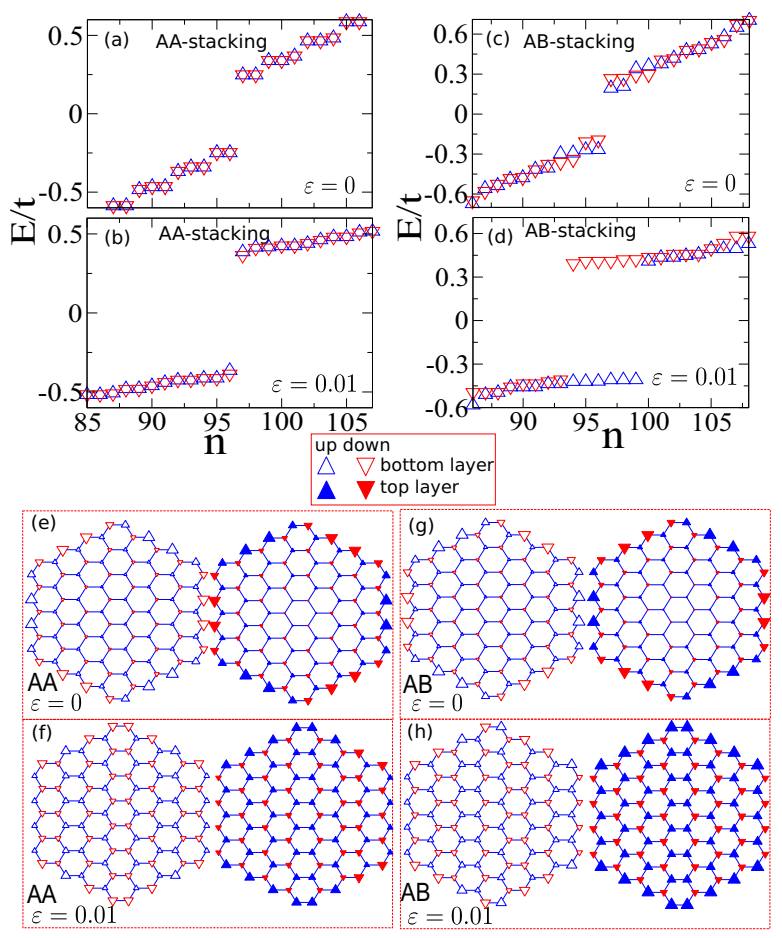

FIG. 9. The same as in Fig. 6, but now for hexagonal BLG QDs with zigzag edges and Coulomb repulsion $U / t=2.5$.
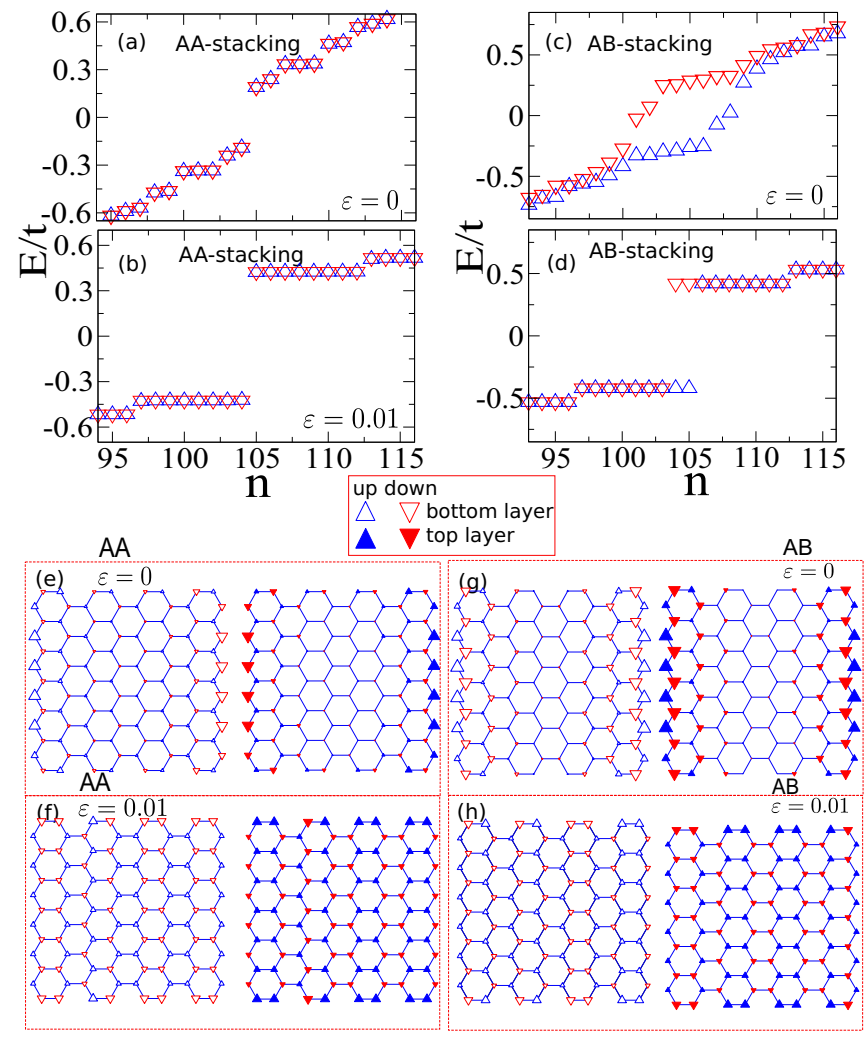

FIG. 10. The same as in Fig. 6, but now for square BLG QDs and Coulomb repulsion $U / t=2.0$.

as observed for square BLG QDs (Fig. 10), but preserving the electron-hole symmetry between spin-up and spin-down electrons. Moreover, for triangular BLG QDs with armchair edges (Fig. 6), one observes that in the absence of strain, for both stacking types, these QDs do not present spin polarization, i.e., they form a nonmagnetic phase related to the absence of zigzag edge states, whereas in the presence of strain, the energy spectrum shows an emergence of magnetism and the energy levels spread around $E=0$.

By analyzing the spin density for all geometries (four bottom panels in Figs. 6 to 10) in the absence [(e) and (g)] and in the presence [(f) and (h)] of strain, it is evident that the main effect of strain on spin density is to break the layer and sublattice symmetries of those BLG QDs. This explains why the maximum of magnetization exhibits two different regimes in Fig. 5. The spin density for the strained BLG QDs above the saturation value $\epsilon>0.7 \times 10^{-4}$ results in an enhancement of $m_{\max }$, as a consequence of its complex ordering that has a mix of ferromagnetic and antiferromagnetic couplings between the two layers. It is important to mention (although not shown here) that the spin densities for the strained BLG QDs below the saturation value (i.e., $\epsilon \leqslant 0.7 \times 10^{-4}$ ) exhibit the same arrangement as the unstrained BLG QDs, explaining why their $m_{\max }$ curve matches the result for $\epsilon=0$ in Fig. 5 .

Furthermore, as seen in the Figs. 6-10, the magnetic moments of the two layers are coupled antiferromagnetically for zigzag-edged and square unstrained BLG QDs, panels (e) and (g) in Figs. 7, 9, and 10, and ferromagnetically for armchair-edged unstrained BLG QDs, panels (e) and (g) in 
Figs. 6 and 8. This means that the local magnetic moments for unstrained BLG QDs exhibit the symmetry $m_{\mathrm{A}, \mathrm{B}}^{\text {bottom }}=m_{\mathrm{A}, \mathrm{B}}^{\text {top }}$ for AA-stacked BLG QDs with armchair edges and AB-stacked BLG QDs with zigzag edges, and $m_{\mathrm{A}, \mathrm{B}}^{\text {bottom }}=-m_{\mathrm{A}, \mathrm{B}}^{\text {top }}$ for ABstacked BLG QDs with armchair edges and AA-stacked BLG QDs with zigzag edges. This is a consequence of the type of edge termination and the fact that the former (latter) BLG QDs possess an imbalanced (balanced) lattice such that the number of atoms in the sublattice A and B in each layer is different (the same). These magnetic orderings of the spin densities are independent of stacking. As shown by the plots of the spin density, one verifies that zigzag-terminated BLG QDs [see Figs. 7(e), 7(g), 9(e), 9(g), 10(e), and 10(g)] display local magnetic moments localized mostly on the zigzag edges, which decrease sharply towards the center of the QD, such that the local spin densities at the bulk sites are in general at least one to two orders of magnitude smaller in comparison to the surface state sites. Also concerning the edge states, it is seen that the local magnetic moments of the zigzag edges are higher close to the middle of the edges and decrease toward the corners, and that for zigzag-edged hexagonal and square BLG QDs we find that the local spins along different edges in each layer point towards opposite directions, since those structures are formed by adjacent edges with atoms belonging to different sublattices and thus these adjacent edges couple antiferromagnetically in the same layer. For instance, for zigzag-edged hexagonal BLG QDs, the spin density for three edges aligns along the up direction, whereas the other three edges point towards the opposite direction, as a consequence of the compensated lattice, while for square BLG QDs, the two zigzag edges are composed by different sublattice types, such that the local spin on these edges point towards opposite directions. For zigzag-edged triangular BLG QDs, where the zigzag edges are formed by atoms that belong to the same sublattice, the surface states are found to couple ferromagnetically in a same layer: it is very clear in Fig. 7(e) that the bottom (top) layer forms a spin-up (spin-down) ferromagnetic phase.

\section{CONCLUSIONS}

In summary, we have investigated the magnetic properties of finite-size BLG QDs with different geometrical shapes: hexagon, triangle, and square, by considering two different types of edges and stacking, namely, zigzag and armchair, and $\mathrm{AA}$ - and $\mathrm{AB}$-stacking layers, respectively, both with and without lattice deformation. We have employed the tightbinding approach coupled with electronic interaction term, that is described by the mean-field approximation of the one-orbital Hubbard model, in order to investigate how the magnetic properties, such as magnetization and the spin energy states, are affected by the presence of uniaxial strain. Our results show that the magnetic properties in general depend on the geometry and not only on the existence of zigzag edges, as also observed for monolayer graphene QDs [27,39]. In the absence of strain, (i) the magnetization as a function of the Hubbard term is not influenced by the dot size for all the considered geometries, except for hexagonal BLG QDs with zigzag edges; and (ii) the magnetization does not depend on the hopping energies $t_{\perp}$ for all geometries, except for BLG QDs with zigzag edges and AA-stacking. This indicates that two decoupled layers in BLG QDs have the same magnetization as the coupled ones. When strain is applied, the nearest-neighbor hopping integrals are naturally modified, which leads to a modification of the local magnetic moments and, consequently, on their magnetic properties. We found that the magnetization is enhanced under uniaxial strain and exhibits two different regimes with respect to the deformation amplitude. We observed, by calculating the local spin density, that this is a consequence of the breaking of the layer and sublattice symmetries in BLG QDs. Furthermore, all discussed results for the strained structures were obtained inside of the linear deformation regime $(\lesssim$ $20 \%$ ) determined by the parameter $\epsilon$, that corresponds to the amplitude of strain, and that obeys the lattice deformation described by Eqs. (7)-(10). The ability to control the magnetic properties by applying strain in BLG QDs makes these systems promising for applications in nanoscale devices and in spintronics.

\section{ACKNOWLEDGMENTS}

This work was financially supported by CNPq, FUNCAP, CAPES Foundation, the Flemish Science Foundation (FWOV1), and the Brazilian Program Science Without Borders (CsF).
[1] K. S. Novoselov, A. K. Geim, S. V. Morozov, D. Jiang, Y. Zhang, S. V. Dubonos, I. V. Grigorieva1, and A. A. Firsov, Science 306, 666 (2004).

[2] A. H. Castro Neto, F. Guinea, N. M. R. Peres, K. S. Novoselov, and A. K. Geim, Rev. Mod. Phys. 81, 109 (2009).

[3] A. V. Rozhkov, G. Giavaras, Y. P. Bliokh, V. Freilikher, and F. Nori, Phys. Rep. 503, 77 (2011).

[4] K. Wakabayashi, Y. Takane, M. Yamamoto, and M. Sigrist, New J. Phys. 11, 095016 (2009).

[5] C. Stampfer, S. Fringes, J. Güttinger, F. Molitor, C. Volk, B. Terrés, J. Dauber, S. Engels, S. Schnez, A. Jacobsen, S. Dröscher, T. Ihn, and K. Ensslin, Front. Phys. 6, 271 (2011).
[6] J. Güttinger, F. Molitor, C. Stampfer, S. Schnez, A. Jacobsen, S. Dröscher, T. Ihn, and K. Ensslin, Rep. Prog. Phys. 75, 126502 (2012).

[7] O. V. Yazyev, Rep. Prog. Phys. 73, 056501 (2010).

[8] H. Abdelsalam, T. Espinosa-Ortega, and I. Luk'yanchuk, Low Temp. Phys. 41, 396 (2015).

[9] D. R. da Costa, Andrey Chaves, M. Zarenia, J. M. Pereira Jr., G. A. Farias, and F. M. Peeters, Phys. Rev. B 89, 075418 (2014).

[10] Z. Z. Zhang, K. Chang, and F. M. Peeters, Phys. Rev. B 77, 235411 (2008).

[11] M. Zarenia, A. Chaves, G. A. Farias, and F. M. Peeters, Phys. Rev. B 84, 245403 (2011) 
[12] H. P. Heiskanen, M. Manninen, and J. Akola, New J. Phys. 10, 103015 (2008).

[13] J. Akola, H. P. Heiskanen, and M. Manninen, Phys. Rev. B 77, 193410 (2008).

[14] D. R. da Costa, M. Zarenia, Andrey Chaves, G. A. Farias, and F. M. Peeters, Phys. Rev. B 92, 115437 (2015).

[15] D. R. da Costa, M. Zarenia, Andrey Chaves, G. A. Farias, and F. M. Peeters, Carbon 78, 392 (2014).

[16] D. R. da Costa, M. Zarenia, Andrey Chaves, G. A. Farias, and F. M. Peeters, Phys. Rev. B 93, 085401 (2016).

[17] D. R. da Costa, M. Zarenia, Andrey Chaves, J. M. Pereira Jr., G. A. Farias, and F. M. Peeters, Phys. Rev. B 94, 035415 (2016).

[18] M. Mirzakhani, M. Zarenia, S. A. Ketabi, D. R. da Costa, and F. M. Peeters, Phys. Rev. B 93, 165410 (2016).

[19] D. Moldovan and F. M. Peeters, Phys. Status Solidi 10, 39 (2016).

[20] M. Wimmer, A. R. Akhmerov, and F. Guinea, Phys. Rev. B 82, 045409 (2010).

[21] M. Ezawa, Phys. Rev. B 76, 245415 (2007).

[22] L. Pisani, J. A. Chan, B. Montanari, and N. M. Harrison, Phys. Rev. B 75, 064418 (2007).

[23] D. Pesin and A. H. MacDonald, Nat. Mater. 11, 409 (2012).

[24] N. O. Weiss, H. Zhou, L. Liao, Y. Liu, S. Jiang, Y. Huang, and X. Duan, Adv. Mater. 24, 5782 (2012).

[25] Y.-W. Son, M. L. Cohen, and S. G. Louie, Nature (London) 444, 347 (2006).

[26] D. Gunlycke, D. A. Areshkin, J. Li, J. W. Mintmire, and C. T. White, Nano Lett. 7, 3608 (2007).

[27] T. Espinosa-Ortega, I. A. Lukyanchuk, and Y. G. Rubo, Phys. Rev. B 87, 205434 (2013).

[28] S. Bhowmick and V. B. Shenoy, J. Chem. Phys. 128, 244717 (2008).

[29] H. Feldner, Z. Y. Meng, A. Honecker, D. Cabra, S. Wessel, and F. F. Assaad, Phys. Rev. B 81, 115416 (2010).

[30] J. Fernández-Rossier and J. J. Palacios, Phys. Rev. Lett. 99, 177204 (2007).

[31] M. Grujić, M. Tadić, and F. M. Peeters, Phys. Rev. B 87, 085434 (2013).

[32] R. Farghadan and A. Saffarzadeh, J. Appl. Phys. 115, 174310 (2014).
[33] A. Zhou and W. Sheng, J. Phys.: Condens. Matter 27, 406002 (2015).

[34] P. Potasz, A. D. Güçlü, O. Voznyy, J. A. Folk, and P. Hawrylak, Phys. Rev. B 83, 174441 (2011).

[35] W.-L. Ma and S.-S. Li, Phys. Rev. B 86, 045449 (2012).

[36] W. Sheng, K. Luo, and A. Zhou, Phys. Rev. B 90, 085406 (2014).

[37] I. Ozfidan, M. Korkusinski, and P. Hawrylak, Phys. Status Solidi RRL 10, 13 (2016).

[38] P. Potasz, A. D. Güçlü, A. Wójs, and P. Hawrylak, Phys. Rev. B 85, 075431 (2012).

[39] J. Viana-Gomes, Vitor M. Pereira, and N. M. R. Peres, Phys. Rev. B 80, 245436 (2009).

[40] S. Cheng, J. Yu, T. Ma, and N. M. R. Peres, Phys. Rev. B 91, 075410 (2015).

[41] F. Qi and G. Jin, J. Appl. Phys. 114, 073509 (2013).

[42] Y. Li, X. Jiang, Z. Liu, and Z. Liu, Nano Res. 3, 545 (2010).

[43] G. Gui, J. Li, and J. Zhong, Phys. Rev. B 78, 075435 (2008).

[44] V. M. Pereira, A. H. Castro Neto, and N. M. R. Peres, Phys. Rev. B 80, 045401 (2009).

[45] R. M. Ribeiro, V. M. Pereira, N. M. R. Peres, P. R. Briddon, and A. H. C. Neto, New J. Phys. 11, 115002 (2009).

[46] B. Roy, F. F. Assaad, and I. F. Herbut, Phys. Rev. X 4, 021042 (2014).

[47] C. Lee, X. Wei, J. W. Kysar, and J. Hone, Science 321, 385 (2008).

[48] A. D. Güçlü, P. Potasz, and P. Hawrylak, Phys. Rev. B 84, 035425 (2011).

[49] E. McCann and M. Koshino, Rep. Prog. Phys. 76, 056503 (2013).

[50] O. V. Yazyev, Phys. Rev. Lett. 101, 037203 (2008).

[51] W. P. Su, J. R. Schrieffer, and A. J. Heeger, Phys. Rev. Lett. 42, 1698 (1979).

[52] H. Thomann, L. R. Dalton, M. Grabowski, and T. C. Clarke, Phys. Rev. B 31, 3141 (1985).

[53] E. H. Lieb, Phys. Rev. Lett. 62, 1201 (1989).

[54] J. Kim, C. Lee, S. Bae, S. J. Kim, K. S. Kim, B. H. Hong, and E. J. Choi, Appl. Phys. Lett. 100, 041910 (2012).

[55] L. Wang and Q. Zhang, Curr. Appl. Phys. 12, 1173 (2012). 